\title{
Dietary carotenoid intake is positively associated with bone density in individuals of the EPIC-Norfolk cohort
}

\author{
R.P.G. Hayhoe ${ }^{1}$, M.A.H. Lentjes ${ }^{2}$, R.N. Luben ${ }^{2}$, K-T. Khaw ${ }^{2}$ and A.A. Welch ${ }^{1}$ \\ ${ }^{1}$ Population Health and Primary Care, Norwich Medical School, University of East Anglia, Norwich, \\ Norfolk NR4 7TJ and ${ }^{2}$ Public Health and Primary Care, University of Cambridge, Cambridgeshire CB1 8RN
}

Osteoporosis is a debilitating disease characterised by low bone density and increased risk of fracture. It is significantly associated with age and is thus an increasing public health concern for our ageing population ${ }^{(1)}$. Nutrition represents an important modifiable factor influencing bone health, with the significance of dietary calcium and vitamin D well established ${ }^{(2)}$. Research has begun to indicate that other nutrients may too be important for bone metabolism, including potassium, magnesium, and vitamin $\mathrm{C}$, found in abundance in fruit and vegetables ${ }^{(3)}$. Fruit and vegetables are also rich sources of carotenoids and limited evidence exists from non-UK studies demonstrating a positive association between specific carotenoid intakes and bone density ${ }^{(4-5)}$, and that high serum carotenoid concentration is associated with reduced risk of osteoporosis ${ }^{(3)}$. The aim of this study was thus to explore the potential association of carotenoid intake with bone density status in a UK population.

Analyses were conducted using a random sample of 4,000 individuals from the EPIC-Norfolk cohort ${ }^{(6)}$ of 25,639 men and women attending an initial health check. Participants completed self-administered health and lifestyle questionnaires and dietary nutrient intake was estimated from 7-day food diaries. At a second health check (mean follow-up 3.6 years) broadband ultrasound attenuation (BUA) of the calcaneus was measured using a CUBA device (McCue Ultrasonics, Winchester). Multivariable regression analysis was used to investigate differences in calcaneal BUA across sex-specific quintiles of dietary carotenoid intake; total carotene, cryptoxanthin, lycopene, and combined lutein and zeaxanthin, were investigated individually. Each fully adjusted model included age, BMI, smoking status, physical activity, family history of osteoporosis, menopausal and HRT status in women, corticosteroid use, calcium intake and supplement use, vitamin D supplement use, and total energy intake. Statistical analyses were performed using STATA (v12; Stata Corp., USA).

Data for the selected variables were available for 968 men and 1360 women. Mean (SD) age at ultrasound measurement was $63 \cdot 0$ (9.3) years for men and $61.7(9 \cdot 2)$ years for women. The majority $(75.4 \%)$ of women were post-menopausal, and $35.3 \%$ were current or former users of HRT. Mean (SD) daily dietary intakes were 2803 (1687) $\mu \mathrm{g}$ carotene, 437 (614) $\mu \mathrm{g}$ cryptoxanthin, 1378 (1644) $\mu \mathrm{g}$ lycopene, and 1101 (834) $\mu \mathrm{g}$ combined lutein and zeaxanthin in men; and 2688 (1486) $\mu \mathrm{g}$ carotene, 461 (610) $\mu \mathrm{g}$ cryptoxanthin, 1282 (1383) $\mu \mathrm{g}$ lycopene, and 1129 (905) $\mu \mathrm{g}$ combined lutein and zeaxanthin in women. Mean (SD) BUA was 89.6 (17.4) dB/MHz for men and $72 \cdot 1(16.5) \mathrm{dB} / \mathrm{MHz}$ for women. Significant positive trends in fully adjusted BUA were apparent across quintiles of total carotene $(p=0.001)$ in women, and combined lutein and zeaxanthin in men $(p=0.03)$ and women $(p<0.001)$. Fully adjusted BUA was $6 \cdot 0 \%$ greater $(p=0.001)$ for total carotene quintile $5 \mathrm{vs}$. quintile 1 in women, and $4.4 \%$ greater $(p=0.03)$ for combined lutein and zeaxanthin quintile $5 \mathrm{vs}$. quintile 1 in men and $6.0 \%(\mathrm{p}=0.001)$ in women.

This study provides novel evidence that dietary carotenoid intake is relevant to bone health in both men and women and thus provides additional support for public health interventions promoting fruit and vegetable consumption. To our knowledge, this is the first time that positive associations between specific carotenoid intake and bone density status have been observed in a UK population cohort. Further investigation into the mechanisms of these associations and the potential protective role of carotenoids against osteoporosis is now required, along with longitudinal study of fracture incidence according to carotenoid intake.

1. Rachner TD, Khosla S \& Hofbauer LC (2011) Lancet 377, 1276-87.

2. Gennari C (2001) Public Health Nutr 4, 547-59.

3. Suguira M, Nakamura M, Ogawa K et al. (2012) PLoS One 7, e52643.

4. Wattanapenpaiboon N, Lukito W, Wahlqvist ML et al. (2003) Asia Pac J Clin Nutr 12, 467-73.

5. Sahni S, Hannan MT, Blumberg J et al. (2009) Am J Clin Nutr 89, 416-24.

6. Day N, Oakes S, Luben R et al. (1999) Br J Cancer 80, 95-103. 